\title{
KCNQ Channels in the Mesolimbic Reward Circuit Regulate Nociception in Chronic Pain in Mice
}

\author{
Hao-Ran Wang ${ }^{1,2,3} \cdot$ Su-Wan Hu ${ }^{1,2} \cdot$ Song Zhang ${ }^{1,2,4} \cdot$ Yu Song ${ }^{1,2} \cdot$ \\ Xiao-Yi Wang ${ }^{1,2} \cdot$ Lei Wang ${ }^{1,2} \cdot$ Yang-Yang $\mathrm{Li}^{1,2} \cdot$ Yu-Mei Yu ${ }^{1,2} \cdot$ He Liu ${ }^{1,2,5}$. \\ Di Liu ${ }^{1,2} \cdot$ Hai-Lei Ding ${ }^{1,2} \cdot$ Jun-Li Cao ${ }^{1,2,5}$
}

Received: 8 May 2020/Accepted: 21 October 2020/Published online: 26 April 2021

(C) The Author(s) 2021

\begin{abstract}
Mesocorticolimbic dopaminergic (DA) neurons have been implicated in regulating nociception in chronic pain, yet the mechanisms are barely understood. Here, we found that chronic constructive injury (CCI) in mice increased the firing activity and decreased the KCNQ channel-mediated M-currents in ventral tegmental area (VTA) DA neurons projecting to the nucleus accumbens (NAc). Chemogenetic inhibition of the VTA-to-NAc DA neurons alleviated CCI-induced thermal nociception. Opposite changes in the firing activity and M-currents were recorded in VTA DA neurons projecting to the medial prefrontal cortex (mPFC) but did not affect nociception. In addition, intra-VTA injection of retigabine, a KCNQ opener, while reversing the changes of the VTA-to-NAc DA neurons, alleviated CCI-induced nociception, and this was abolished by injecting exogenous BDNF into the NAc.
\end{abstract}

Hao-Ran Wang, Su-Wan Hu and Song Zhang have contributed equally.

Jun-Li Cao

caoj10310@aliyun.com

1 Jiangsu Province Key Laboratory of Anesthesiology, Xuzhou Medical University, Xuzhou 221004, China

2 Jiangsu Province Key Laboratory of Anesthesia and Analgesia Application Technology, Xuzhou Medical University, Xuzhou 221004, China

3 Department of Anesthesiology, The First Affiliated Hospital of Nanjing Medical University (Jiangsu Province Hospital), Nanjing 210029, China

4 Department of Anesthesiology, Renji Hospital, Shanghai Jiaotong University School of Medicine, Shanghai 200000, China

5 Department of Anesthesiology, The Affiliated Hospital of Xuzhou Medical University, Xuzhou 221002, China
Taken together, these findings highlight a vital role of KCNQ channel-mediated modulation of mesolimbic DA activity in regulating thermal nociception in the chronic pain state.

Keywords Nociception - Mesocorticolimbic system · Ventral tegmental area - Brain-derived neurotrophic factor $\cdot$ KCNQ $\cdot$ Retigabine $\cdot$ Chronic neuropathic pain

\section{Introduction}

Chronic pain has become an enormous health care issue, affecting $>15 \%$ of the world population [1, 2]. The literature suggests that the mesolimbic reward system plays a critical role in regulating chronic pain [3-6]. The ventral tegmental area (VTA), a core part of the mesolimbic reward system, contains predominantly dopaminergic (DA) neurons [7]. The VTA sends DA projections to both limbic and cortical areas and forms two important circuits: the mesolimbic circuit (VTA to the nucleus accumbens, VTA-to-NAc) and the mesocortical circuit (VTA to the medial prefrontal cortex, VTA-to-mPFC) $[8,9]$.

Both increases and decreases in the firing activity of VTA DA neurons have been reported in chronic pain states [10-12]. Theses discrepancies may be explained by the intricate heterogeneity of the different DA projection neurons. There is evidence that the firing activity of VTA DA neurons modulates the functional output of the reward system through its downstream targets [13, 14]. As such, the VTA-to-NAc and VTA-to-mPFC DA neurons may undergo differential adaptations in chronic pain states. Furthermore, the NAc and the mPFC, at the single-brainregion level, have been studied extensively for their roles in the processing of nociception under physiological and 
pathological pain conditions [11, 15-18]. Therefore, we speculated that the mesolimbic and mesocortical circuits play different roles in regulating nociceptive responses in chronic pain states.

The roles of the mesolimbic DA neurons in regulating nociception are strongly associated with their neuronal activity [19]. We previously reported that the increased firing activity of the VTA-to-NAc DA neurons mediates the thermal hyperalgesia induced by chronic constriction injury (CCI) of the sciatic nerve by releasing brain-derived neurotrophic factor (BDNF) into the NAc [19]. However, the mechanisms underlying the changes of DA neuronal activity remain largely unknown.

$\mathrm{K}^{+}$voltage-gated channel subfamily Q (KCNQ) channels are widely expressed in neurons and have been implicated in pain [20-24]. Several studies have shown that KCNQ channels are involved in the modulation of hyperalgesia by regulating neuronal activity in peripheral nerve $[20,21]$. A recent report demonstrated that overexpression of KCNQ channels normalizes the hyperactivity of VTA DA neurons [25]. These findings led us to hypothesize that that KCNQ channels of the VTA DA neurons might be involved in the regulation of nociception in chronic pain states.

Using electrophysiological, chemogenetics, and pharmacological approaches, we explored the roles of the VTAto-NAc and VTA-to-mPFC DA neurons in an animal model of CCI-induced neuropathic pain and further investigated the underlying cellular mechanism.

\section{Materials and Methods}

\section{Animals}

Male C57BL/6J mice aged 7-8 weeks were provided by the Experimental Animal Center of Xuzhou Medical University and were acclimated to the housing facility for 1 week prior to experiments. These mice were grouphoused (5 per cage) and maintained on a 12-h light/dark cycle with controlled humidity and temperature, and ad libitum access to food and water. All experiments were performed during the light cycle and all the behavioral tests were conducted by double-blind randomization in a silent room. All procedures followed the recommendations of the National Institutes of Health Guide for Care and Use of Laboratory Animals as well as the Committee for Research and Ethical Issues of the International Association for the Study of Pain.

\section{Surgery}

CCI surgery was performed following a method described previously [26]. Briefly, mice were anesthetized with $1 \%$ pentobarbital sodium $(40 \mathrm{mg} / \mathrm{kg}$, i.p.) and the skin on the lateral surface of the left thigh was incised. The left sciatic nerve was exposed at mid-thigh level and a unilateral constriction injury just proximal to the trifurcation was performed with three loose ligatures using 4-0 silk (spaced at $1-\mathrm{mm}$ intervals). In sham-operated animals, the nerve was exposed but not ligated. The incision was closed in layers, and the wound was treated with topical penicillin.

\section{Stereotaxic Injections}

Mice were anesthetized with $1 \%$ pentobarbital sodium (40 $\mathrm{mg} / \mathrm{kg}$, i.p.) and then placed in a stereotaxic apparatus (RWD, China). The cranium was exposed with the periosteum removed by $3 \%$ hydrogen peroxide. To label the projection-specific VTA DA neurons, lumafluor $(0.5$ $\mu \mathrm{L}$, Lumafluor Inc., Durham, NC) was injected either into the NAc (in $\mathrm{mm}$ ): anterior/posterior (AP) +1.40 ; medial/ lateral (ML), +0.60 ; dorsal/ventral (DV), -4.70 or into the mPFC (in mm): AP, +1.95; ML, +0.27; DV, -2.00 . Retigabine $(20 \mu \mathrm{mol} / \mathrm{L}, \mathrm{MB} 1200$, Melone Pharmaceutical Co., Ltd., China) was injected into the VTA (in mm): AP, $-3.30 ; \mathrm{ML},+1.05 ; \mathrm{DV},-4.60 ; 7^{\circ}$, in a volume of $0.3 \mu \mathrm{L}$ to investigate the effect of retigabine on behavior associated with neuropathic pain. The viruses were from BrainVTA (Wuhan, China). To evaluate the expression of KCNQ2 and KCNQ3 in VTA-to-NAc and VTA-tomPFC DA neurons, rAAV-TH-NLS-Cre-WPRE-pA $(0.2$ $\mu \mathrm{L}, 2 / \mathrm{R}, 5.65 \times 10^{12}$ genomic copies per $\left.\mathrm{mL}\right)$ and $\mathrm{rAAV}$ Efl $\alpha$-DIO-EGFP-WPRE-pA $\left(0.2 \mu \mathrm{L}, 2 / 9,6.86 \times 10^{12}\right.$ genomic copies per $\mathrm{mL}$ ) were respectively injected into the NAc or mPFC and the VTA. To modulate projectionspecific VTA DA neurons by chemogenetics, rAAV-THNLS-Cre-WPRE-pA $\left(0.2 \mu \mathrm{L}, 2 / \mathrm{R}, 5.65 \times 10^{12}\right.$ genomic copies per $\mathrm{mL}$ ) was injected into the NAc or $\mathrm{mPFC}$, while rAAV-Efl $\alpha$-DIO-hM4D(Gi)-mCherry-WPRE-pA $(0.2 \mu \mathrm{L}$, $2 / 9,5.67 \times 10^{12}$ genomic copies per $\mathrm{mL}$ ) or rAAV-Efl $\alpha$ DIO-hM3D(Gq)-mCherry-WPRE-pA $(0.2 \mu \mathrm{L}, 2 / 9,5.65 \times$ $10^{12}$ genomic copies per $\mathrm{mL}$ ) was injected into the VTA contralateral to the CCI surgery. To overexpress KCNQ2 in projection-specific DA neurons, rAAV-TH-NLS-CreWPRE-pA $\left(0.2 \mu \mathrm{L}, 2 / \mathrm{R}, 5.65 \times 10^{12}\right.$ genomic copies per $\mathrm{mL}$ ) was injected into the NAc or mPFC, and rAAV-CMVDIO-KCNQ2-EGFP $\left(0.2 \mu \mathrm{L}, 2 / \mathrm{R}, 5.65 \times 10^{12}\right.$ genomic copies per $\mathrm{mL}$ ) into the VTA. To elucidate the role of BDNF signaling in retigabine-induced behavioral outcomes in the mesocorticolimbic system, $0.2 \mu \mathrm{L}$ BDNF (10 ng, B3795; Sigma-Aldrich) was injected into the NAc or mPFC. All the reagents were microinjected using a 
Hamilton syringe needle (33-gauge) at $0.1 \mu \mathrm{L} / \mathrm{min}$ with a pump (Harvard Apparatus).

\section{Measurement of Thermal Nociception}

The Hargreaves test [27] was performed to evaluate the thermal nociceptive response by using an IITC plantar analgesia meter (IITC Life Science, Inc.). In brief, mice were placed in plastic cages on a glass platform and allowed to acclimatize for $30 \mathrm{~min}$ before testing. A radiant heat source was positioned directly beneath the glass and focused on the plantar surface of the left hind paw. The nociceptive endpoint in the radiant heat test was the lifting or licking of the hind paw. The time from onset of the radiant heat to the reaction was considered as the paw withdrawal latency (PWL). The radiant heat intensity was adjusted at the beginning of the experiment to obtain a basal PWL of 12-15 s and the cutoff time for exposure was set at $25 \mathrm{~s}$ to prevent tissue damage. The measurements were repeated five times at 5-min intervals and the average of the five measurements was calculated. For the chemogenetic-mediated behavioral test, the animals were injected intraperitoneally with clozapine-N-oxide (CNO; $1 \mathrm{mg} / \mathrm{kg}$, dissolved in saline; HY-17366, MCE) $30 \mathrm{~min}$ prior to the test.

\section{Ex-vivo Electrophysiological Recordings}

All recordings were carried out blind to the experimental conditions of behavioral, drug, and viral treatments. Mice were anesthetized with isofluorane and perfused immediately with ice-cold aCSF (artificial cerebrospinal fluid) for 40-60 s, which contained (in mmol/L): $128 \mathrm{NaCl}, 3 \mathrm{KCl}$, $1.25 \mathrm{NaH}_{2} \mathrm{PO}_{4}, 10$ D-glucose, $24 \mathrm{NaHCO}_{3}, 2 \mathrm{CaCl}_{2}$, and $2 \mathrm{MgCl}_{2}$ (oxygenated with $95 \% \mathrm{O}_{2}$ and $5 \% \mathrm{CO}_{2}$, pH 7.4, 295-305 mOsm). Acute brain slices containing VTA DA neurons were cut on a microslicer (DTK-1000, Ted Pella) in ice-cold sucrose aCSF, in which the $\mathrm{NaCl}$ was fully replaced with $254 \mathrm{mmol} / \mathrm{L}$ sucrose and saturated by $95 \%$ $\mathrm{O}_{2}$ and $5 \% \mathrm{CO}_{2}$. Slices were maintained in holding chambers with aCSF for $1 \mathrm{~h}$ recovery at $37^{\circ} \mathrm{C}$. For experiments, slices were transferred to the recording chamber and perfused $(2-2.5 \mathrm{~mL} / \mathrm{min})$ with oxygenated aCSF at $35^{\circ} \mathrm{C}$.

Patch pipettes (3-5 M $\Omega$ ) for whole-cell current-clamp, voltage-clamp, and cell-attached recordings were filled with internal solution containing the following (in $\mathrm{mmol} /$ L): 115 potassium gluconate, $20 \mathrm{KCl}, 1.5 \mathrm{MgCl}_{2}, 10$ phosphocreatine, 10 HEPES, 2 magnesium ATP, and 0.5 GTP (pH 7.2, 285 mOsm). VTA DA neurons were identified by their location and infrared differential interference contrast microscopy and recordings were made from lumafluor-positive neurons for projection-specific recordings.

Cell-attached recordings were used for measurements of the spontaneous activity of VTA DA neurons. Signals were band-pass filtered at $300 \mathrm{~Hz}-1 \mathrm{kHz}$ to identify DA neurons and were then Bessel filtered at $10 \mathrm{kHz}$ (gain 50) using a Multiclamp 700B amplifier (Molecular Devices). For chemogenetics validations, the spontaneous activity of VTA DA neurons was recorded continuously before, during, and after CNO perfusion $(5 \mathrm{mmol} / \mathrm{L})$.

To measure M-current, whole-cell recordings were carried out in voltage-clamp mode. The neurons were recorded in the presence of $1 \mu \mathrm{mol} / \mathrm{L}$ tetrodotoxin (TTX; Alomone Labs), at a holding potential of $-20 \mathrm{mV}$, and 1-s repolarizing steps were then applied to $-40 \mathrm{mV}$. To measure the intrinsic membrane properties of VTA DA neurons, whole-cell recordings were carried out in currentclamp mode and spikes were induced by $100-p A$ current injection.

Retigabine $(10 \mu \mathrm{mol} / \mathrm{L}, \mathrm{MB} 1200$, Melone Pharmaceutical Co., Ltd., China) was used to investigate the role of KCNQ channels in regulating the activity of VTA DA neurons. Series resistance was monitored during all recordings. Data were acquired and analyzed using a Digidata 1440 A digitizer and pClamp 10.2 (Molecular Devices).

\section{Immunohistochemistry}

Under general anesthesia with $1 \%$ pentobarbital sodium $(40 \mathrm{mg} / \mathrm{Kg}$, i.p. $)$, the mice were intracardially perfused with $30 \mathrm{~mL}$ cold PBS (pH 7.4) and $30 \mathrm{~mL} 4 \%$ paraformaldehyde. The brains were removed and post-fixed at $4{ }^{\circ} \mathrm{C}$ overnight, then kept in 30\% sucrose for 2 days. Coronal sections $(30 \mu \mathrm{m})$ were cut on a freezing microtome (VT1000S, Leica Microsystems). VTA sections were directly imaged for lumafluor, EGFP, and mCherry expression on a laser scanning confocal microscope (FluoView FV1000; Olympus).

For immunofluorescence staining, the sections were blocked for $1 \mathrm{~h}$ with $3 \%$ donkey serum and $0.25 \%$ Triton $\mathrm{X}-100$ in PBS at room temperature. Then they were incubated with the primary antibodies rabbit anti-Kv7.2 (KCNQ2, 1:100; APC-050, Alomone Labs), rabbit antiKv7.3 (KCNQ3, 1:100; APC-051, Alomone Labs), and mouse anti-tyrosine hydroxylase (1:600, 318; Millipore), at $4^{\circ} \mathrm{C}$ overnight. The sections were then incubated for $2 \mathrm{~h}$ at room temperature with the secondary antibodies Alexa Fluor 488 anti-rabbit (A-11034), AlexaFluor 594 antimouse (A-11032), and AlexaFluor 488 anti-mouse (A32723) (1:200, Molecular Probes), and rinsed three times with PBS before mounting. Afterwards, the sections were visualized under an Olympus confocal microscope. 


\section{Western Blot}

NAc and mPFC tissue blocks were harvested with 12-gauge punches and then sonicated in $30 \mu \mathrm{L}$ homogenization buffer containing $320 \mathrm{mmol} / \mathrm{L}$ sucrose, $5 \mathrm{nmol} / \mathrm{L}$ HEPES, $1 \%$ sodium dodecyl sulfate (v/v), phosphatase inhibitor cocktails I and II (Sigma-Aldrich), and protease inhibitors (Roche Diagnostics). Protein concentrations were determined with a DC protein assay (Bio-Rad). Proteins (20-40 $\mu \mathrm{g}$ protein per lane) were electrophoresed in $10 \%$ SDS-PAGE gel and transferred onto PVDF membranes. The membranes were incubated at $4{ }^{\circ} \mathrm{C}$ overnight with the primary rabbit anti-BDNF $(1: 1000$, sc546, Santa Cruz) and mouse anti-GAPDH (1:1000, E021010, Earthox) antibodies. The membranes were rinsed and then incubated for $2 \mathrm{~h}$ at room temperature with the secondary antibodies conjugated to alkaline phosphatase (1:500; Santa Cruz). The immune complexes were detected with a BCIP/NBT kit (Beyotime). Blots were analyzed with Photoshop software (Adobe Systems, Inc.), and the gray-scale values of protein bands were normalized to those of GAPDH.

\section{Statistical Analysis}

Data are presented as mean \pm SEM. Statistical analyses were performed with GraphPad Prism 6.0 software (Graph Pad Software, Inc.). Differences among three or more groups were compared with one-way ANOVA followed by Tukey's post hoc test. The unpaired $t$ test was used if only two groups were compared. The significance of any difference in thermal PWLs in the behavioral tests was assessed with a two-way ANOVA with repeated measures and Bonferroni's post hoc test. All $P$ values given are based on two-tailed tests. For all analyses, $P<0.05$ was considered to be statistically significant.

\section{Results}

\section{Changes of Firing Rates in Mesocorticolimbic DA Neurons Under Chronic Pain}

PWLs were used to assess nociceptive responses [27]. CCI mice exhibited a significant decrease in the PWLs of the affected hind paws, which lasted for at least 14 days (Fig. 1A, B). To investigate the firing activity of the VTAto-NAc and VTA-to-mPFC DA neurons in a state of CCIinduced chronic neuropathic pain, we injected the retrograde tracer lumafluor into the NAc or mPFC 7 days before CCI surgery to label projection-specific VTA neurons. Cell-attached electrophysiological recordings were performed in these labeled neurons, and the DA neurons were identified by their waveforms under signal-filtered condition (see Methods) (Fig. 1C). The ex-vivo recordings showed that CCI induced a significant increase in the firing activity in the contralateral, but not the ipsilateral, VTA-toNAc DA neurons (Fig. 1D). In contrast, the firing activity of contralateral VTA-to-mPFC DA neurons was dramatically decreased (Fig. 1E). These data indicate that the VTA-to-NAc and VTA-to-mPFC DA neurons respond differentially to CCI-induced neuropathic pain.

\section{Roles of the Mesocorticolimbic Circuits in Regula- tion of CCI-Induced Thermal Nociception}

To answer the question whether these activity changes of the mesocorticolimbic DA neurons are implicated in regulation of thermal nociception in CCI-induced neuropathic pain, we first chemogenetically decreased the activity of VTA-to-NAc DA neurons by injection of a retrograde AAV, rAAV-TH-NLS-Cre-WAPE-pA, into the NAc, and a cre-dependent AAV, rAAV-Efla-DIOhM4D(Gi)-mCherry-WPRE-pA, into the VTA (Fig. 2A, B). The expression of hM4Di in VTA DA neurons was confirmed by co-localization of TH and mCherry (Fig. 2B) and its function was validated as a decreased firing rate of mCherry-labeled VTA DA neurons in acute slices induced by CNO perfusion ( $5 \mathrm{mmol} / \mathrm{L}$ ) (Fig. 2C). CCI surgery was conducted 2 weeks following the microinjections, and 7 days after that, PWLs were measured. CCI mice with CNO injection (1 mg/kg, i.p.) showed increased PWLs (Fig. 2D), suggesting that the chemogenetic inhibition of VTA-toNAc DA neurons reversed CCI-induced thermal hyperalgesia.

Next, we tested whether the decreased firing activity of the VTA-to-mPFC DA neurons contributed to the thermal nociception in CCI mice. To upregulate the firing activity in these neurons, we injected rAAV-TH-NLS-Cre-WAPE$\mathrm{pA}$ into the mPFC, and rAAV-Efl $\alpha$-DIO-hM3D $(\mathrm{Gq})$ mCherry-WPRE-pA into the VTA (Fig. 2A, E). The viral expression and its function were confirmed by immunofluorescence staining and cell-attached recordings (Fig. 2E, F). Unlike inhibition of the VTA-to-NAc DA neurons, this chemogenetic activation by $\mathrm{CNO}$ injection did not change the PWLs in CCI mice (Fig. 2G). These results suggest an involvement of the VTA-to-NAc, but not the VTA-tomPFC DA neurons, in regulating thermal nociception under a state of chronic neuropathic pain.

\section{Changes of M-current $\left(I_{M}\right)$ in Mesocorticolimbic DA Neurons Under Chronic Pain}

$I_{\mathrm{M}}$, a voltage-gated $\mathrm{K}^{+}$current, is involved in the physiological and pathological modulation of neuronal activity [28-30]. It is mediated by KCNQ-type $\mathrm{K}^{+}$ 
A

B

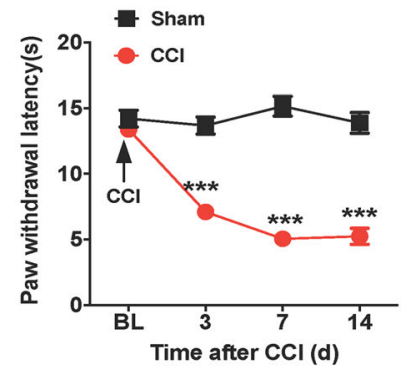

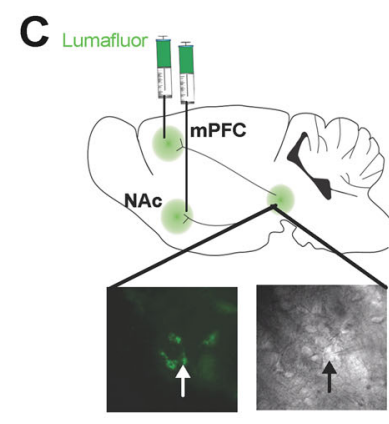

D

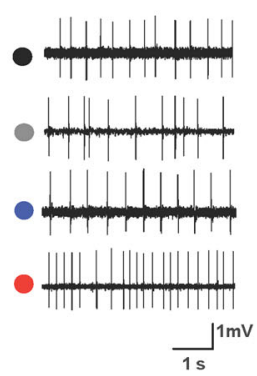

Thermal nociceptive test

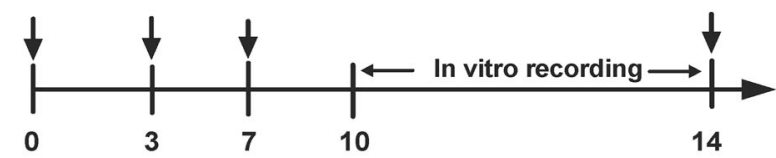

E

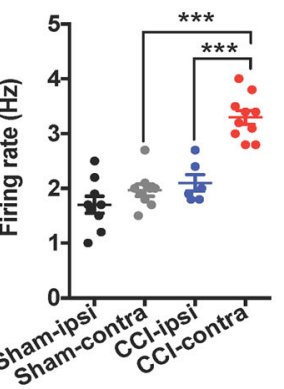

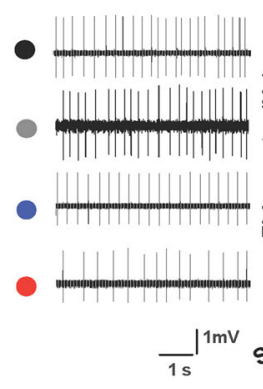

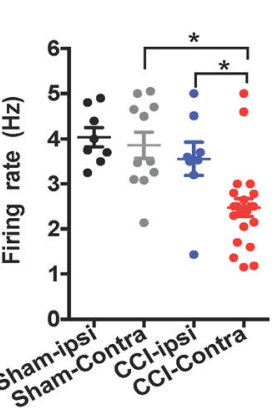

Fig. 1 CCI induces different changes in the firing rates of VTA-toNAc and VTA-to-mPFC DA neurons. A Experimental timeline. B Summary results showing that PWLs are lower on day 3, 7, and 14 after CCI surgery than in sham mice $(n=7$ mice/group; $* * * P<0.001$, two-way ANOVA with repeated measures and Bonferroni's posttest). C Schematic of retrograde lumafluor injection into the NAc or $\mathrm{mPFC}$ and labeling lumafluor in the VTA (scale bar, $50 \mu \mathrm{m}$ ). D Sample traces and statistics of cell-attached recordings showing

channels [28, 31] and thus measurement of $I_{M}$ reflects the function of KCNQ channels. We used the retrograde tracer lumafluor to label VTA-to-NAc and VTA-to-mPFC neurons and performed ex-vivo whole-cell recordings to measure $I_{M}$ in these labeled cells from CCI mice. The results showed that $\mathrm{CCI}$ mice exhibited decreased $\mathrm{M}$-current density in the contralateral VTA-to-NAc DA neurons (Fig. 3A) and increased density the contralateral VTA-tomPFC DA neurons (Fig. 3B), suggesting that CCI differentially modulates the KCNQ channels of mesocorticolimbic DA neurons, and thus may lead to the opposite changes of firing activity in the VTA-to-NAc and VTA-tomPFC DA neurons.

\section{Location of KCNQ2/3 Channels in Mesocorticolim- bic DA Neurons}

Immunohistochemical studies have shown that KCNQ2 and KCNQ3 channels are present in VTA neurons [30, 32-34]. Thus, we investigated the location of KCNQ2/3 channels in mesocorticolimbic DA neurons. To do this, we labeled the two DA populations by microinjection of a retrograde AAV, rAAV-TH-NLS-Cre-WAPE$\mathrm{pA}$, into the NAc or mPFC, and a cre-dependent AAV, rAAV-Efl $\alpha$-DIO-EGFP-WPRE-pA, into the VTA. The EGFP- and TH-positive cells were concerned as the that CCI induces an increase in the firing rates of contralateral VTAto-NAc DA neurons $(n=9-12$ cells $/ 3-4$ mice/group; $* * * P<0.001 v s$ sham-contra and CCI-ipsi groups, one-way ANOVA with Tukey's post-test). E Sample traces and statistics of cell-attached recordings showing that $\mathrm{CCI}$ induces a decrease in the firing rates of contralateral VTA-to-mPFC DA neurons $(n=10-22$ cells $/ 3-5$ mice/group; $* P$ $<0.05$ vs sham-contra and CCI-ipsi groups, one-way ANOVA with Tukey's post-test). Error bars show mean and SEM.

putative circuit-specific DA populations. KCNQ2-labeled cells constituted $91.69 \%$ of VTA-to-NAc DA neurons (Fig. 4A, C), and $96.23 \%$ of VTA-to-mPFC neurons (Fig. 4B, D). Meanwhile, $81.65 \%$ of the VTA-to-NAc DA neurons (Fig. 4E, G) and $82.66 \%$ of the VTA-to-mPFC population (Fig. 4F, H) were KCNQ3-positive.

We further analyzed the proportions of KCNQ2- or KCNQ3-positive cells in VTA-to-NAc or VTA-to-mPFC DA neurons. No significant differences were seen between the CCI mice and the sham counterparts (Fig. 4A-H). These data suggest no quantitative changes of the DA neurons that contain KCNQ2/KCNQ3 channels in CCIinduced chronic pain. It is worth noting that this result does not deny differential expression of KCNQ2/KCNQ3 channels in the two VTA DA projections in chronic neuropathic pain.

\section{Roles of Circuit-Specific KCNQ2 Overexpression in Regulating Thermal Nociception}

Changes in both KCNQ expression and channel status may contribute to $I_{\mathrm{M}}$ alterations. To explore the roles of KCNQ channels in regulating thermal nociception in a chronic pain state, we overexpressed KCNQ2 in each of the two specific DA populations by injecting rAAV-TH-cre into the NAc or mPFC, and rAAV-CMV-DIO-KCNQ2-EGFP into 


\section{A}

NAc or mPFC: rAAV-TH-Cre

VTA:DIO-hM4D-mcherry or DIO-hM3D-mcherry

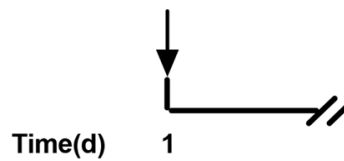

1. $\mathrm{CCl}$ Surgery

2. Epression validatation

Time(d)

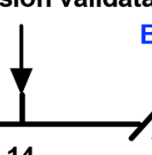

Inhibitoin of VTA $\rightarrow$ NAC DA Circuitry

B
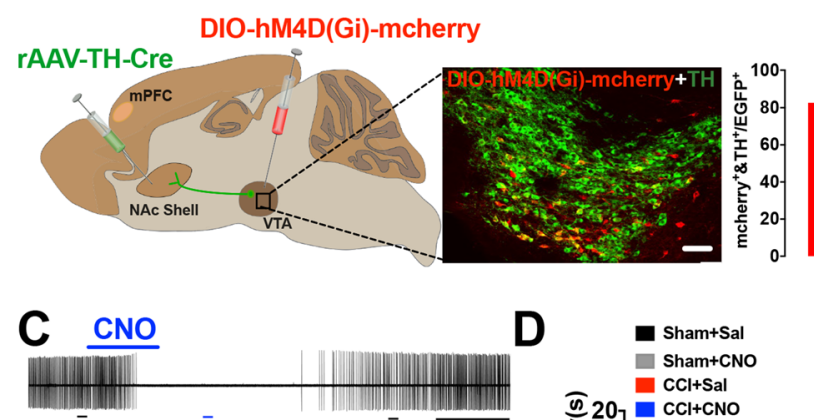

$\overline{\mathbf{a}}$

$\overline{\mathbf{b}}$

a:

b: -
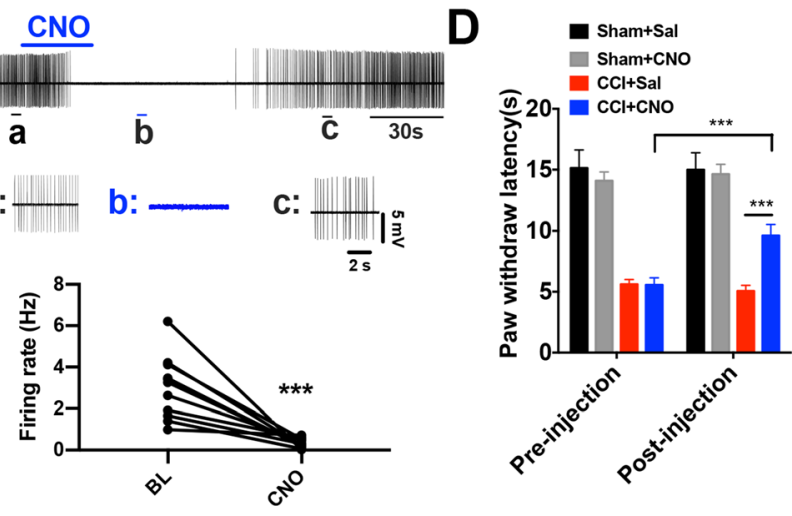

Fig. 2 The VTA-to-NAc, but not the VTA-to-mPFC DA circuit, regulates thermal nociception in CCI mice. A Experimental timeline. B Schematic of viral injections of retrograde rAAV-TH-NLS-CreWAPE-pA into the NAc and cre-dependent rAAV-Efl $\alpha$-DIOhM4D(Gi)-mCherry-WPRE-pA into the VTA; representative confocal image showing co-expression of hM4Di expression (red) in VTA DA neurons (green) (scale bar, $200 \mu \mathrm{m}$ ). C Sample traces and statistics from VTA slices showing that the firing activity of putative VTA-to-NAc DA neurons are inhibited by CNO perfusion $(n=11$ cells from 6 mice; $* * * P<0.001$, paired $t$ test). D Summary data showing that CCI mice with CNO injection exhibit alleviated nociceptive responses compared to mice with saline injection $(\mathrm{n}=$ 8-9 mice/group; $* * * P<0.001$ vs pre-CNO injection and $\mathrm{CCI}+\mathrm{Sal}$

the VTA (Fig. 5A, B, F). After validation of viral expression (Fig. 5B, F), whole-cell recordings confirmed that this projection-selective overexpression of KCNQ2 increased the M-current density (Fig. 5C) and decreased the firing activity (Fig. 5D). Behavioral results showed that KCNQ2 overexpression in the VTA-to-NAc DA neurons reversed the established thermal hyperalgesia in CCI mice as evidenced by the increased PWLs (Fig. 5E). This reversal was not seen in the mice with KCNQ2 overexpression in the VTA-to-mPFC DA neurons (Fig. 5G), These results indicate that functional modulation of KCNQ2 channels in the VTA-to-NAc, rather than the
E

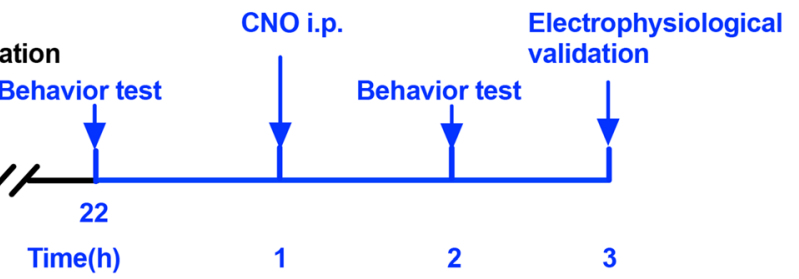

Activation of VTA $\rightarrow$ mPFC DA Circuitry

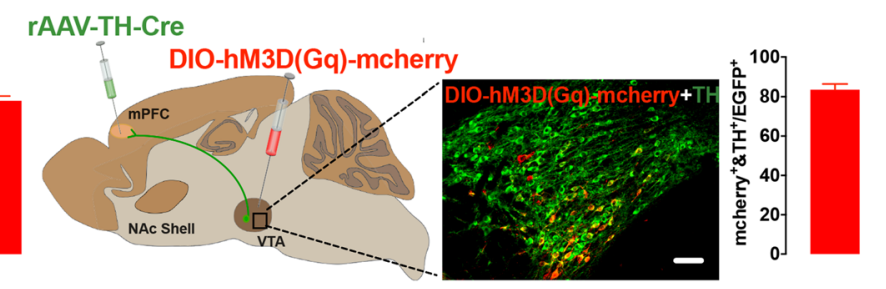

$\mathbf{F}$
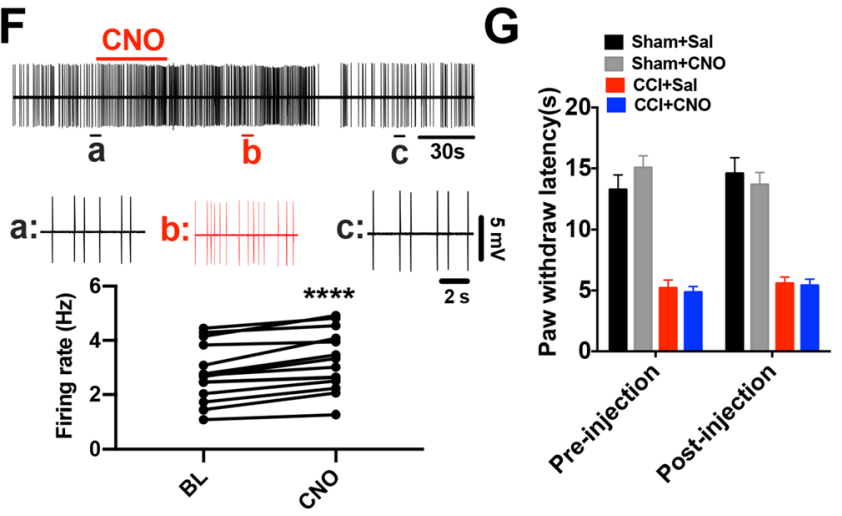

groups, two-way ANOVA with repeated measures and Bonferroni's post-test). E Schematic of viral injections of a retrograde rAAV-THNLS-Cre-WAPE-pA into the mPFC and cre-dependent rAAV-Efl $\alpha$ DIO-hM3D(Gq)-mCherry-WPRE-pA into the VTA; representative confocal image showing co-expression of hM3Dq expression (red) in VTA DA neurons (green) (scale bar, $200 \mu \mathrm{m}$ ). F Sample traces and statistics from VTA slices showing that the firing of putative VTA-tomPFC DA neurons is increased by CNO perfusion ( $n=16$ cells from 6 mice; $* * * * P<0.0001$, Wilcoxon test). G Summary data showing that CNO injection does not change the PWLs of CCI mice $(n=6-8$ mice/group; two-way ANOVA with repeated measures and Bonferroni's post-test). Error bars show the mean and SEM.

VTA-to-mPFC circuit contributes to regulating thermal nociception in chronic neuropathic pain.

\section{Relief of CCI-Induced Thermal Nociception by Retigabine}

Given that the functional status of KCNQ channels may also affect $I_{\mathrm{M}}$, we further investigated their effects on thermal nociception by activating KCNQ channels. The KCNQ opener retigabine, an FDA-approved agent for anticonvulsant therapy [35-37], was used here. The results from ex-vivo whole-cell recordings in VTA slices showed 
A

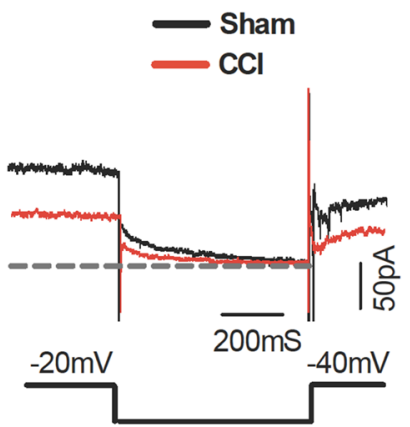

Fig. $3 \mathrm{CCI}$ induces different changes in the $I_{M}$ of VTA-to-NAc and VTA-to-mPFC DA neurons. A Sample traces and statistics from VTA-to-NAc DA neurons showing decreased M-current density in CCI mice $(n=11$ cells $/ 3-4$ mice/group; $* * P<0.01$, unpaired $t$ test).

A

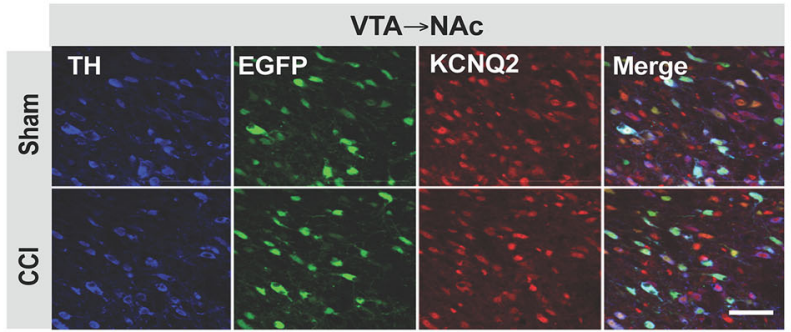

B

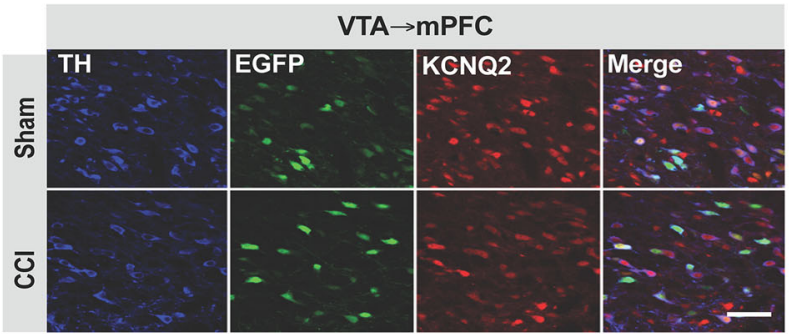

C

VTA $\rightarrow$ NAC

\section{KCNQ2- \\ KCNQ2}

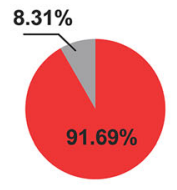

Percentage of total $\mathrm{EGFP}^{+} \& \mathrm{TH}^{+}$cells

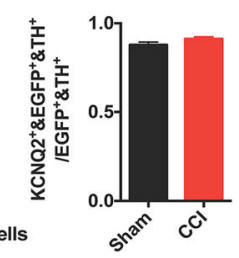

D VTA $\rightarrow$ mPFC
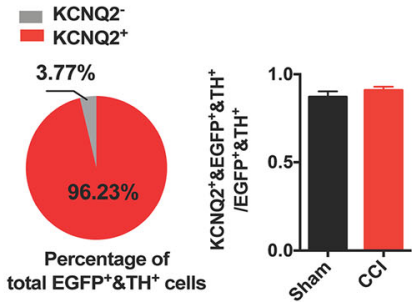

Fig. 4 Location of KCNQ2 and KCNQ3 in VTA-to-NAc and VTAto-mPFC DA neurons. A Confocal images showing the expression of KCNQ2 (red) in putative VTA-to-NAc DA neurons (blue and green) (scale bar, $50 \mu \mathrm{m})$. B Confocal images showing the expression of KCNQ2 (red) in the putative VTA-to-mPFC DA neurons (blue and green) (scale bar, $50 \mu \mathrm{m}$ ). C Statistics for the percentage of KCNQ2positive cells in putative VTA-to-NAc DA neurons; the sham and CCI mice did not differ (2-3 sections/mouse from 3 mice; unpaired $t$ test). D Statistics for the percentage of KCNQ2-positive cells in putative VTA-to-mPFC DA neurons; the sham and CCI mice did not differ (2-3 sections/mouse from 3 mice; unpaired $t$ test). E Confocal images
B $\quad$ VTA $\rightarrow$ mPFC neurons
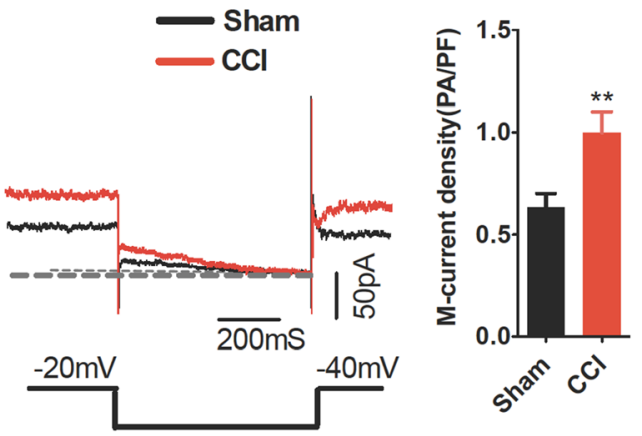

B Sample traces and statistics from VTA-to-mPFC DA neurons showing increased M-currents in CCI mice $(n=11-15$ cells $/ 3-5$ mice/group; $* * P<0.01 \mathrm{vs}$ sham group, unpaired $t$ test).

E

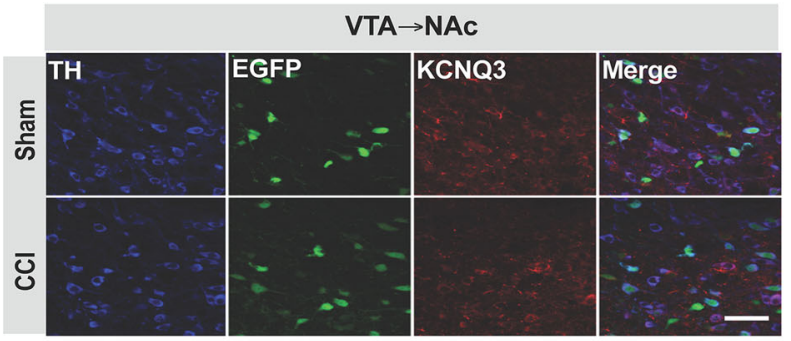

$\mathbf{F}$

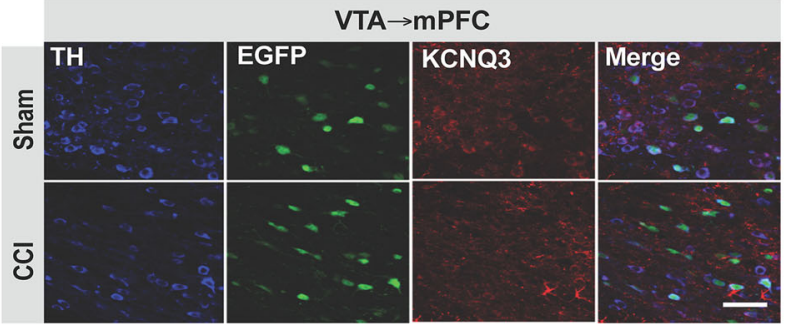

G

VTA $\rightarrow$ NAC

H

VTA $\rightarrow$ mPFC
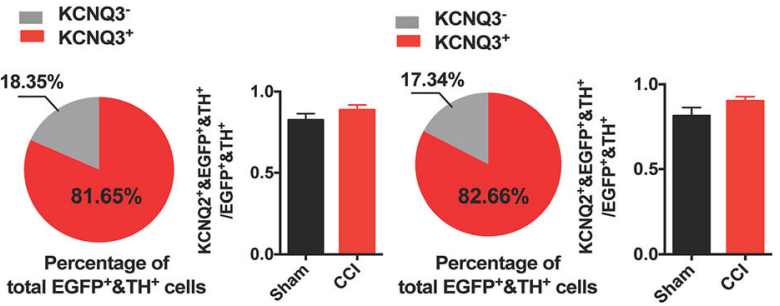

showing the expression of KCNQ3 (red) in putative VTA-to-NAc DA neurons (blue and green) (scale bar, $50 \mu \mathrm{m}$ ). F Confocal images showing the expression of KCNQ3 (red) in putative VTA-to-mPFC DA neurons (blue and green) (scale bar, $50 \mu \mathrm{m}$ ). G Statistics for the percentage of KCNQ3-positive cells in putative VTA-to-NAc DA neurons; the sham and CCI mice did not differ (2-3 sections/mouse from 3 mice; unpaired $t$ test). $\mathbf{H}$ Statistics for the percentage of KCNQ3-positive cells in putative VTA-to-mPFC DA neurons; the sham and CCI mice did not differ (2-3 sections/mouse from 3 mice; unpaired $t$ test). Error bars show the mean and SEM. 

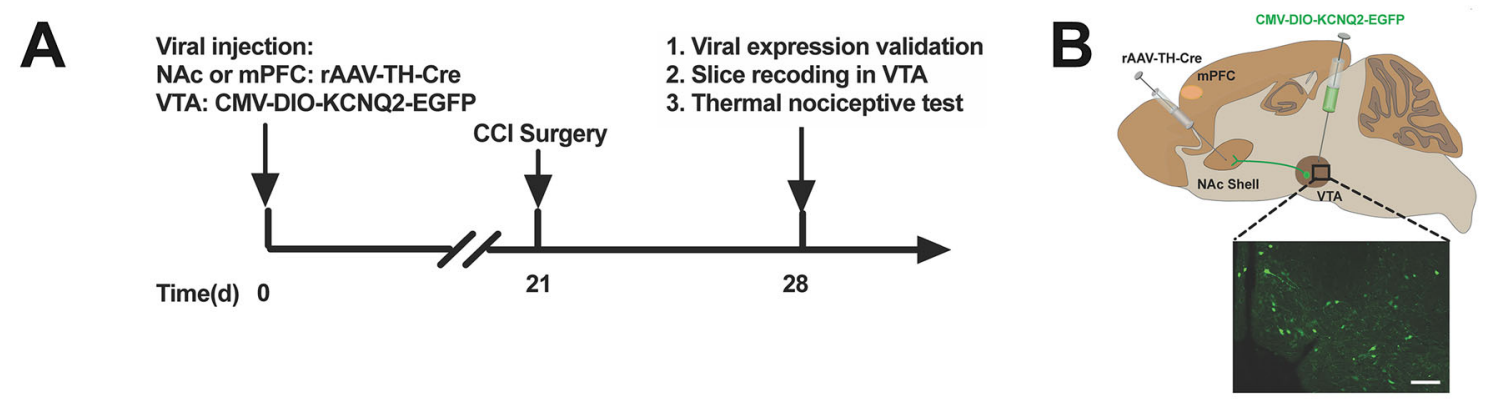

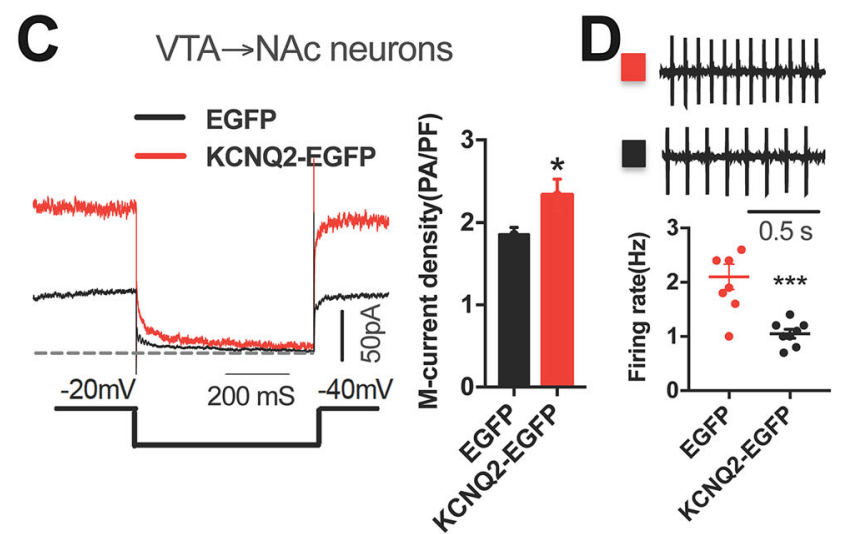

Fig. 5 KCNQ2 overexpression in the VTA-to-NAc, but not the VTAto-mPFC DA neurons relieves thermal nociception in CCI mice. A Experimental timeline. B Schematic of viral injections of a retrograde rAAV-TH-NLS-Cre-WAPE-pA into the NAc, and a credependent rAAV-CMV-DIO-KCNQ2-EGFP-WPRE-pA into the VTA; representative confocal image showing KCNQ2-EGFP expression (green) in the VTA (scale bar, $100 \mu \mathrm{m}$ ). C Sample traces and statistics from VTA-to-NAc DA neurons showing increased M-current density in CCI mice with KCNQ2 overexpression in VTA-toNAc DA neurons $(n=10-22$ cells $/ 3-5$ mice/group; $* P<0.05$, unpaired $t$ test). D Sample traces and summary showing that the firing rate of VTA-to-NAc DA neurons was decreased in CCI mice with

that perfusion of retigabine significantly increased the M-current density and membrane potential (Fig. 6A-F), and decreased the firing rate (Fig. $6 \mathrm{G}, \mathrm{H}$ ) in both VTA-toNAc and VTA-to-mPFC DA neurons as compared with the vehicle controls, suggesting its non-selective effects on the mesocorticolimbic circuits. Furthermore, intra-VTA injection of retigabine $(10 \mu \mathrm{mol} / \mathrm{L}, 0.15 \mu \mathrm{L})$ produced a clear anti-nociceptive response as evidenced by the increased PWLs (Fig. 6I). Combined with our previous finding, we suggest that retigabine exerts its anti-nociceptive effects by decreasing the activity of the VTA-to-NAc DA neurons by activating KCNQ channels.

\section{Mesocorticolimbic BDNF Signaling in the Anti-no- ciceptive Effect of Retigabine}

BDNF plays important roles in regulating synaptic plasticity and neuronal survival $[38,39]$. Previous studies have
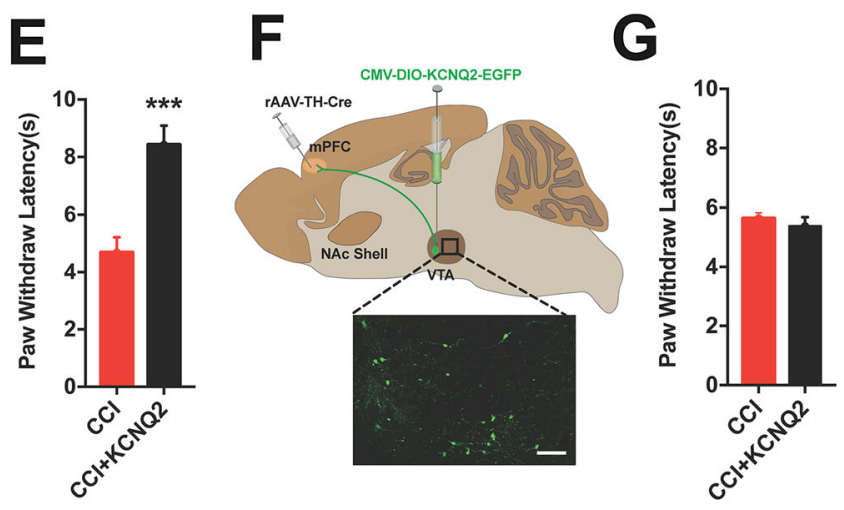

KCNQ2 overexpression in these neurons $(n=10-22$ cells $/ 3-5$ mice/group; $* * * P<0.001$, unpaired $t$ test). E Summary showing PWLs are increased after KCNQ2 overexpression in VTA-to-NAc DA neurons ( $n=8 \mathrm{mice} /$ group; $* * * P<0.001$, unpaired $t$ test). F Schematic of viral injections of retrograde rAAV-TH-NLS-CreWAPE-pA into the mPFC, and cre-dependent rAAV-CMV-DIOKCNQ2-EGFP-WPRE-pA into the VTA; representative confocal image showing KCNQ2-EGFP expression (green) in the VTA (scale bar, $100 \mu \mathrm{m}$ ). G Summary data showing that KCNQ2 overexpression in the VTA-to-mPFC DA neurons does not change the PWLs in CCI mice ( $n=8$ mice/group; unpaired $t$ test). Error bars show the mean and SEM.

suggested that VTA DA neurons release BDNF into the targeted areas in an activity-dependent manner [38, 40]. Our recent work showed that BDNF in the VTA-to-NAc circuit is increased, thus mediating thermal nociception in mice with CCI-induced neuropathic pain [19]. Therefore, we asked whether BDNF signaling in the VTA-NAc circuit is involved in the anti-nociceptive effect of retigabine. Western blot assays showed that the BDNF protein levels in the NAc were drastically increased in CCI mice and this increase was reversed by intra-VTA injection of retigabine (Fig. 7A), suggesting that intra-VTA injection of retigabine decreased BDNF levels in the NAc under chronic pain. Furthermore, exogenous administration of BDNF into the NAc abolished the anti-nociceptive effect of intra-VTA retigabine injection in CCI mice (Fig. 7C). Unlike that in the NAc, CCI induced a significant decrease of BDNF protein levels in the mPFC (Fig. 7B). Intra-VTA injection of retigabine further lowered the BDNF levels in the $\mathrm{mPFC}$ 

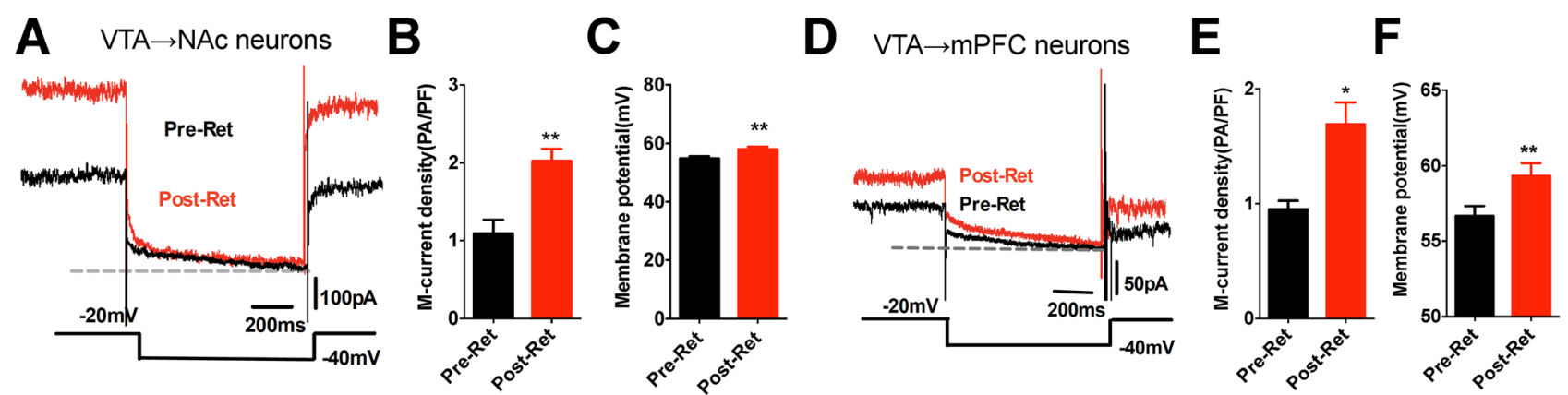
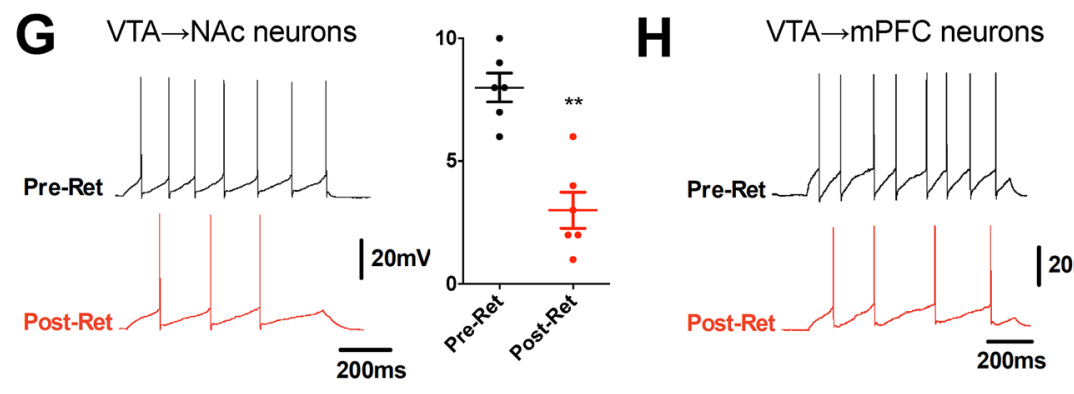

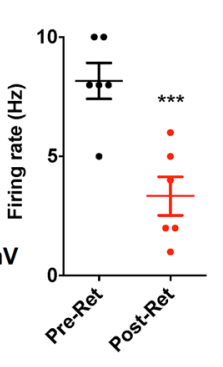

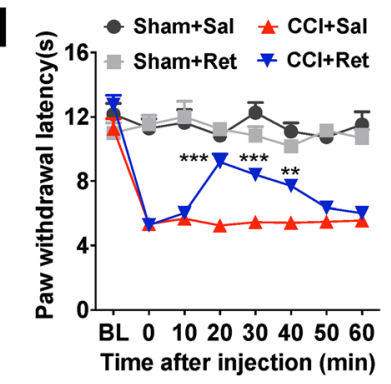

Fig. 6 Intra-VTA injection of retigabine reduces nociceptive responses in CCI mice. A-C Sample traces (A) and quantitative data $(\mathbf{B}, \mathbf{C})$ showing that retigabine increases the M-current density and membrane potential in VTA-to-NAc DA neurons $(n=12-16$ cells/ 3-4 mice/group; $* * P<0.01$, unpaired $t$ test). D-F Sample traces (D) and quantitative data $(\mathbf{E}, \mathbf{F})$ showing that retigabine increase the M-current density and membrane potential in VTA-to-mPFC DA neurons $(n=12-16$ cells $/ 3-4$ mice/group, $* P<0.05, * * P<0.01$, unpaired $t$ test). G Sample traces and statistics from whole-cell recordings (100 pA current injection) showing decreased activity of

in CCI mice (Fig. 7B). Exogenous BDNF injected into the mPFC did not alter the anti-nociceptive effect induced by intra-VTA retigabine injection (Fig. 7D). These data imply a potential role of BDNF signaling in the VTA-to-NAc DA circuit in the anti-nociceptive effect of retigabine on chronic neuropathic pain.

\section{Discussion}

Our current results demonstrate that: (1) CCI differentially modulated the firing activity of the VTA-to-NAc and VTAto-mPFC DA circuits, and of the two, the former is involved in thermal nociception regulation; (2) both KCNQ2 overexpression in the VTA-to-NAc DA neurons and intra-VTA injection of the KCNQ opener retigabine corrected mesolimbic KCNQ channel-mediated M-currents and DA neuronal activity, and relieved CCI-induced thermal nociception; and (3) BDNF signaling in the VTA-to-NAc DA circuit may be involved in the antinociceptive effect of retigabine. These findings reveal a novel KCNQ channel-based ionic and circuit mechanism
VTA-to-NAc DA neurons $(n=6$ cells $/ 3$ mice/group; $* * P<0.01$, unpaired $t$ test). H Sample traces and statistics from whole-cell recordings (100 pA current injection) showing decreased activity of VTA-to-mPFC DA neurons ( $n=6$ cells/3 mice/group; $* * P<0.01$, unpaired $t$ test). I Summary data showing that intra-VTA injection of retigabine increases PWLs in CCI mice $(n=6$ mice/group; ***P $<0.001, * * P<0.01$ vs CCI+Sal group, two-way ANOVA with repeated measures and Bonferroni's post-test). Error bars show the mean and SEM.

for the regulatory roles of mesocorticolimbic circuits in chronic neuropathic pain-induced thermal nociception.

VTA DA neurons are a heterogeneous population divided into several subgroups in terms of their targets $[13,41]$. Among them, the VTA-to-NAc and the VTA-tomPFC DA neurons have been shown to have distinct properties: for example, these subpopulations have different firing properties [42, 43]. Specifically, the VTA-tomPFC DA neurons discharge faster than the VTA-to-NAc DA neurons [42, 43], as confirmed by our current results (Fig. 1C-E).

Pain is a stressor, causing multiple adaptive changes in the brain [44, 45]. A growing body of literature suggests that the mesocorticolimbic DA circuits are implicated in chronic pain $[3,6,19,25,46]$. Similar stress can induce different firing alterations in these projection-specific VTA DA neurons [47]. For instance, recent studies have shown that the VTA-to-NAc and VTA-to-mPFC DA neurons exhibit distinct properties and actions in regulating stressrelated depression [48-51]. Likewise, our current study reports such heterogeneity in CCI-induced chronic neuropathic pain. In line with our previous study [19], the current results suggest that the mesolimbic DA circuit is required 
Fig. 7 BDNF signaling in the VTA-to-NAc DA circuit is involved in the anti-nociceptive effect of retigabine. A Typical bands and summary showing upregulated BDNF protein levels in the NAc of CCI mice $(n=3 ; * P<0.05$ vs sham + Sal group, one-way ANOVA with Tukey's post-test); this was reversed by retigabine injection into the VTA $\left(n=3 ;{ }^{*} P<0.05\right.$ vs $\mathrm{CCI}+\mathrm{Sal}$ group, one-way ANOVA with Tukey's posttest). B Typical bands and summary showing downregulated BDNF protein levels in the mPFC of CCI mice $(n=3 ; * P$ $<0.05 v s$ sham + Sal group, oneway ANOVA with Tukey's post-test); this was further reduced by retigabine injection into the VTA $(n=3 ; * P<0.05$ vs CCI+Sal group, one-way ANOVA with Tukey's posttest). C Summary data showing that intra-NAc injection of exogenous BDNF abolishes the anti-nociceptive effect of intraVTA retigabine injection in CCI mice $(n=6$ mice/group; $* * P$ $<0.01$ vs Sal (NAc) + Ret (VTA) group, two-way ANOVA with repeated measures and Bonferroni's posttest). D Summary data showing that intra-mPFC injection of exogenous BDNF does not alter the anti-nociceptive effect of retigabine in CCI mice $(n=6$ mice/group; two-way ANOVA with repeated measures and Bonferroni's post-test). Error bars show the mean and SEM.

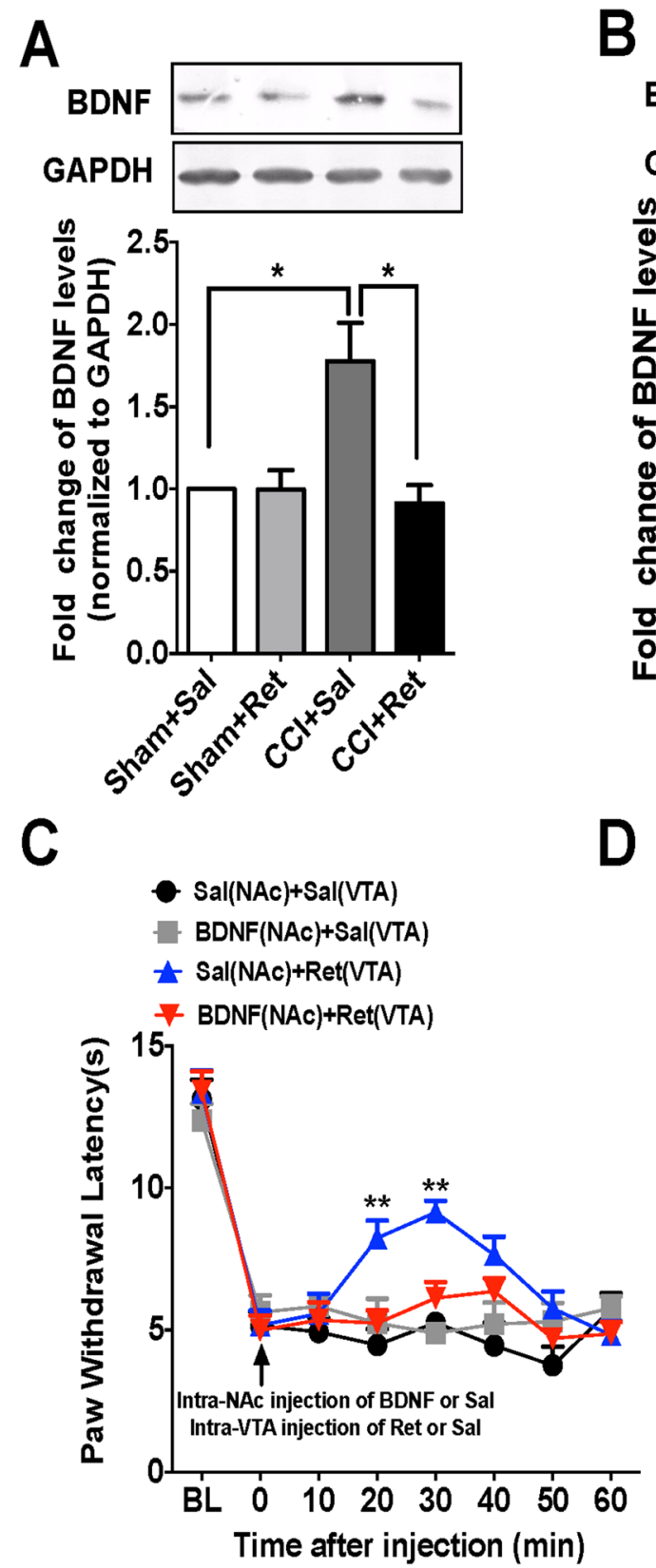

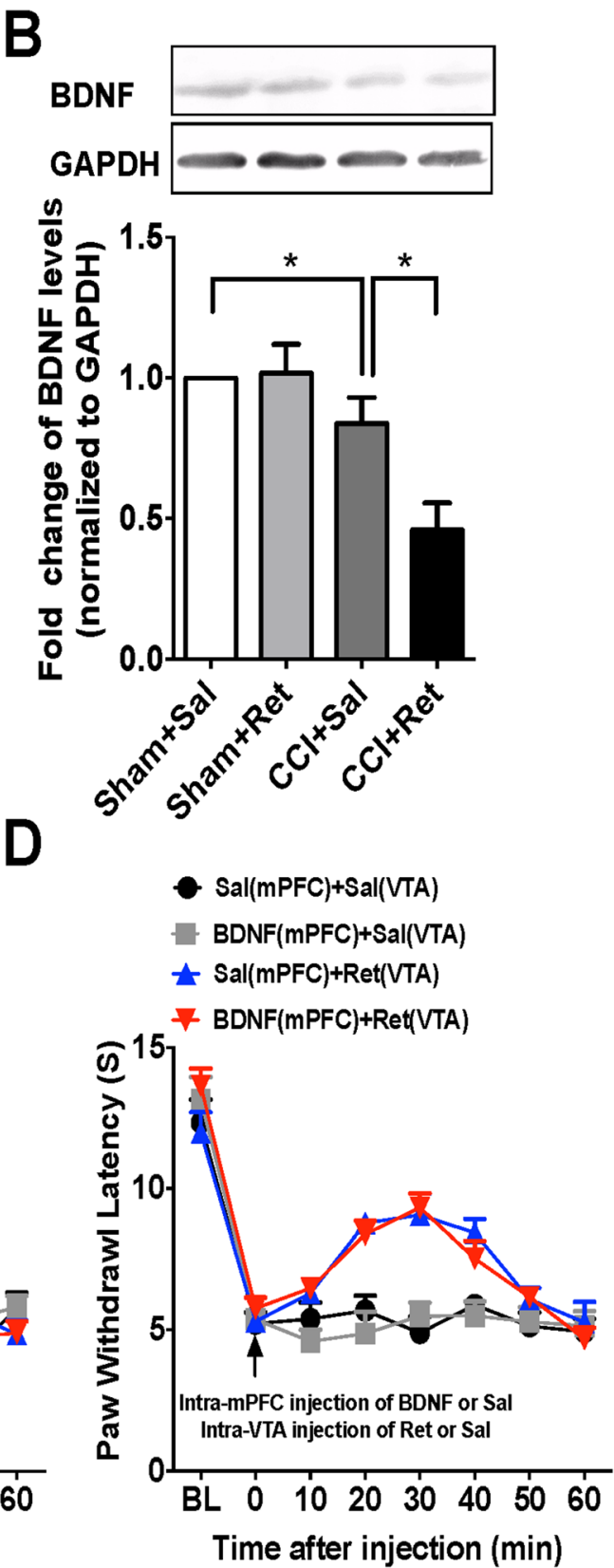

for the regulation of thermal nociception in a state of chronic pain (Fig. 1, 2). Although the firing activity of VTA-to-mPFC DA neurons was decreased by CCI (Fig. 1E), activation of these neurons did not change the thermal nociceptive threshold (Fig. 2E-G). This result does not support an involvement of VTA-to-mPFC DA neurons in regulating the thermal nociception underlying chronic pain. It should be noted that the function of the decreased activity in the mesocortical DA neurons remains unknown. Based on recent studies [50, 52], we speculate that this adaptive change may be involved in regulating chronic pain-related negative emotions and cognitive impairments.
It has been shown that KCNQ channels serve as a brake to suppress abnormal ectopic discharges of neurons and control neuronal hyperexcitability [25, 28, 33]. These channels and related $\mathrm{M}$-currents set the resting membrane potential [53], and exert an inhibitory effect on neuronal discharges in brain slice, spinal cord, and dorsal root ganglion neurons [20, 25, 54]. Structurally, KCNQ channels consist of five subunits as homo- or hetero-tetramers [28, 31, 33]. Among them, KCNQ2/3 channels are well recognized to form the molecular basis for M-currents [31], and have been found to affect DA neuronal activity within the VTA [32, 55]. It is known that the KCNQ2/3 heteromers exhibit an 11-fold larger current than the 
KCNQ2 or KCNQ3 homomers [56]. Based on their abundant distribution (Fig. 4), we speculate that the majority of KCNQ2 and KCNQ3 channels form heteromers in the VTA. Furthermore, both KCNQ2 overexpression and microinjection of the KCNQ opener retigabine corrected the CCI-induced changes of M-currents and firing activity in the VTA-to-NAc DA neurons and reversed thermal hyperalgesia. These data support the hypothesis that chronic neuropathic pain may heterogeneously modulate the expression and/or functional status of KCNQ channels in mesocorticolimbic DA circuits, resulting in the distinct firing properties. Although KCNQ4 and KCNQ5 may contribute to M-currents likewise, we did not test their roles due to their locations $[57,58]$.

The functional heterogeneity of the mesocorticolimbic DA circuits is largely dependent on changes of the firing activity in the VTA DA subpopulations [50, 51], and yet various ion channels expressed within these neurons play an important role as intrinsic factors in regulating firing activity [59]. Studies have shown that hyperpolarizationactivated cation channel-mediated currents $\left(I_{\mathrm{h}}\right)$ provide an excitatory driving force [60-62] and $\mathrm{K}^{+}$channel-mediated currents provide an inhibitory driving force in VTA DA neurons [30, 63], and the balance between them is essential for normal function of the mesocorticolimbic DA circuits [55, 63, 64]. External stimuli may shift this balance towards an excitatory or inhibitory state in different projecting circuits [55], and then result in heterogeneous functional phenotypes. The ion channel-related heterogeneous alterations in mesocorticolimbic DA circuits have been well studied in the social defeat stress-induced depression model $[25,55]$. We report similar ion channel-mediated functional heterogeneity of the mesocorticolimbic DA circuits in chronic pain processing. In detail, KCNQ channel-mediated M-type $\mathrm{K}^{+}$currents, as an inhibitory driving force $[30,63]$, display heterogeneous alterations in the mesocorticolimbic DA circuits in mice with CCI-induced neuropathic pain (Fig. 3), which may result in changes of the firing activity of DA neurons.

Our previous study has shown that the VTA-to-NAc DA neurons exhibit an increase of the excitatory driving force $I_{\mathrm{h}}$ current in CCI mice and pharmacologically inhibiting VTA DA neuron firing with $I_{\mathrm{h}}$ blockers reverses CCIinduced NAc neuronal activation and thermal hyperalgesia in CCI mice [19]. Thus, we speculate that long-lasting nociceptive inputs with chronic pain shift the balance towards an excitatory state in the VTA-to-NAc DA circuit via modulating $I_{\mathrm{h}}$ and $\mathrm{M}$-type $\mathrm{K}^{+}$currents and therefore increase the firing activity of these neurons. These studies strongly support the conclusion that the intrinsic firing activity of VTA DA neurons serves as an important mechanism underlying the adaptations involved in chronic pain, and may provide valuable therapeutic targets for chronic pain.

Retigabine is an FDA-approved anticonvulsant drug for partial epilepsies. It reduces neuronal excitability in animals and humans by enhancing $I_{M}$ via the activation of KCNQ channels [35]. Recent studies have also implicated this drug in treating lateral sclerosis [65], alcohol addiction [66], chronic pain [20-22], and depression [25]. Administration of retigabine has been reported to reverse mechanical and thermal hypersensitivity in inflammatory, neuropathic, and cancer pain and chemotherapy-induced peripheral neuropathy models [20-22, 24]. Retigabine exerts an analgesic effect through peripheral mechanisms including inhibiting the excitability of nociceptive neurons and C-type nerve fibers [20, 24]. Studies have also reported that intra-thalamic or intra-cerebral ventricular injection of retigabine produce an analgesic effect $[67,68]$, suggesting that a supraspinal mechanism may be involved in the antinociceptive effect of retigabine. In the present study, intraVTA injection of retigabine alleviated CCI-induced thermal nociception, in parallel with increased M-currents and decreased firing activity in VTA DA neurons (Fig. 6). Combined with the finding that the adaptive changes in the VTA-to-NAc DA neurons contribute to CCI-induced thermal nociception, we speculate that correction of the KCNQ-mediated firing activity in VTA-to-NAc DA neurons may underlie the anti-nociceptive effect of retigabine.

Previous studies have demonstrated that BDNF is a critical nociceptive modulator in the mesolimbic reward circuit $[19,51]$. There is a strong association between VTA DA neuronal firing rates and BDNF levels in the target regions [19, 38, 40]. We provide evidence that intra-VTA injection of retigabine corrected BDNF protein levels in the NAc in CCI mice (Fig. 7A, B). As BDNF levels in the NAc may reflect the VTA DA neuronal activity, this change in BDNF levels is in line with the finding that retigabine decreased the firing activity of the VTA DA neurons in CCI mice (Fig. 6G, H). In addition, exogenous BDNF in the NAc abolished the effect of retigabine on thermal nociception in CCI mice (Fig. 7C). These results reflect a possible role of BDNF signaling of the VTA-to-NAc DA circuit in the anti-nociceptive effect of retigabine. It is worth noting that retigabine similarly changed the BDNF levels in the mPFC, whereas exogenous BDNF delivered to the mPFC had no effect on thermal nociception (Fig. 7B, D). This point needs future investigations on the part played by the retigabine-induced decrease in BDNF levels in the mPFC.

Since pain is a distressing experience with emotional and cognitive components, a limitation of this study is that we only focused on thermal pain sensation. Whether and how this system is involved in processing pain-related negative emotions and cognition impairments remain to be 
explored. Limitations in viral expression may also occur due to nonspecific expression and the influence of injection sites.

In summary, our study reveals the functional heterogeneity of the mesocorticolimbic DA circuits in regulating thermal nociception in the chronic pain state, and unmasks a novel mechanism by which mesolimbic KCNQ channels regulate thermal nociception by modulating the VTA-toNAc DA neuronal firing activity.

Acknowledgements This work was supported by grants from the National Natural Science Foundation of China (31771161, $81720108013,81230025,81200859$ and 81801096), Key Project of Nature Science Foundation of Jiangsu Education Department (17KJA320005), Natural Science Foundation of Jiangsu Province (BK20171159), the Qing-Lan Project of Jiangsu, the Six Talent Summit Project of Jiangsu, the 333 High-level Personnel Training Project of Jiangsu, the Priority Academic Program Development of Jiangsu Higher Education Institutions, the Jiangsu Provincial Special Program of Medical Science (BL2014029), and the Postgraduate Research \& Practice Innovation Program of Jiangsu Province (KYCX19-2241 and KYCX20_2449), China.

Conflict of interest The authors declare no competing interests.

Open Access This article is licensed under a Creative Commons Attribution 4.0 International License, which permits use, sharing, adaptation, distribution and reproduction in any medium or format, as long as you give appropriate credit to the original author(s) and the source, provide a link to the Creative Commons licence, and indicate if changes were made. The images or other third party material in this article are included in the article's Creative Commons licence, unless indicated otherwise in a credit line to the material. If material is not included in the article's Creative Commons licence and your intended use is not permitted by statutory regulation or exceeds the permitted use, you will need to obtain permission directly from the copyright holder. To view a copy of this licence, visit http://creativecommons. org/licenses/by/4.0/.

\section{References}

1. Baliki MN, Apkarian AV. Nociception, pain, negative moods, and behavior selection. Neuron 2015, 87: 474-491.

2. Murray CJ, Lopez AD. Measuring the global burden of disease. N Engl J Med 2013, 369: 448-457.

3. Mitsi V, Zachariou V. Modulation of pain, nociception, and analgesia by the brain reward center. Neuroscience 2016, 338 : 81-92.

4. Navratilova E, Atcherley CW, Porreca F. Brain circuits encoding reward from pain relief. Trends Neurosci 2015, 38: 741-750.

5. Navratilova E, Porreca F. Reward and motivation in pain and pain relief. Nat Neurosci 2014, 17: 1304-1312.

6. Zhang S, Yang XN, Zang T, Luo J, Pan Z, Wang L. Astroglial MicroRNA-219-5p in the Ventral Tegmental Area Regulates Nociception in Rats. Anesthesiology 2017, 127: 548-564.

7. Bjorklund A, Dunnett SB. Dopamine neuron systems in the brain: an update. Trends Neurosci 2007, 30: 194-202.

8. Hauser TU, Eldar E, Dolan RJ. Separate mesocortical and mesolimbic pathways encode effort and reward learning signals. Proc Natl Acad Sci USA 2017, 114: E7395-E7404.
9. Lammel S, Ion DI, Roeper J, Malenka RC. Projection-specific modulation of dopamine neuron synapses by aversive and rewarding stimuli. Neuron 2011, 70: 855-862.

10. Fu B, Wen SN, Wang B, Wang K, Zhang JY, Weng XC, et al. Gabapentin regulates dopaminergic neuron firing and theta oscillation in the ventral tegmental area to reverse depressionlike behavior in chronic neuropathic pain state. J Pain Res 2018, 11: 2247-2256.

11. Ren W, Centeno MV, Berger S, Wu Y, Na X, Liu X, et al. The indirect pathway of the nucleus accumbens shell amplifies neuropathic pain. Nat Neurosci 2016, 19: 220-222.

12. Watanabe M, Narita M, Hamada Y, Yamashita A, Tamura H, Ikegami $\mathrm{D}$, et al. Activation of ventral tegmental area dopaminergic neurons reverses pathological allodynia resulting from nerve injury or bone cancer. Mol Pain 2018, 14: 1744806918756406.

13. Borgkvist A, Mrejeru A, Sulzer D. Multiple personalities in the ventral tegmental area. Neuron 2011, 70: 803-805.

14. Roeper J. Dissecting the diversity of midbrain dopamine neurons. Trends Neurosci 2013, 36: 336-342.

15. Schwartz N, Temkin P, Jurado S, Lim BK, Heifets BD, Polepalli JS, et al. Chronic pain. Decreased motivation during chronic pain requires long-term depression in the nucleus accumbens. Science 2014, 345: 535-542.

16. Zhang Z, Gadotti VM, Chen L, Souza IA, Stemkowski PL, Zamponi GW. Role of prelimbic GABAergic circuits in sensory and emotional aspects of neuropathic pain. Cell Rep 2015, 12: 752-759.

17. Ong WY, Stohler CS, Herr DR. Role of the prefrontal cortex in pain processing. Mol Neurobiol 2019, 56: 1137-1166.

18. Xu L, Wan Y, Ma L, Zheng J, Han B, Liu F-Y, et al. A contextbased analgesia model in rats: involvement of prefrontal cortex. Neurosci Bull 2018, 34: 1047-1057.

19. Zhang H, Qian YL, Li C, Liu D, Wang L, Wang XY, et al. Brainderived neurotrophic factor in the mesolimbic reward circuitry mediates nociception in chronic neuropathic pain. Biol Psychiatry 2017, 82: 608-618.

20. Passmore GM, Selyanko AA, Mistry M, Al-Qatari M, Marsh SJ, Matthews EA, et al. $\mathrm{KCNQ} / \mathrm{M}$ currents in sensory neurons: significance for pain therapy. J Neurosci 2003, 23: 7227-7236.

21. Wu Z, Li L, Xie F, Du J, Zuo Y, Frost JA, et al. Activation of KCNQ channels suppresses spontaneous activity in dorsal root ganglion neurons and reduces chronic pain after spinal Ccord injury. J Neurotrauma 2017, 34: 1260-1270.

22. Blackburn-Munro G, Jensen BS. The anticonvulsant retigabine attenuates nociceptive behaviours in rat models of persistent and neuropathic pain. Eur J Pharmacol 2003, 460: 109-116.

23. Tsantoulas C, McMahon SB. Opening paths to novel analgesics: the role of potassium channels in chronic pain. Trends Neurosci 2014, 37: 146-158.

24. Zheng Q, Fang D, Liu M, Cai J, Wan Y, Han JS, et al. Suppression of KCNQ/M (Kv7) potassium channels in dorsal root ganglion neurons contributes to the development of bone cancer pain in a rat model. Pain 2013, 154: 434-448.

25. Friedman AK, Juarez B, Ku SM, Zhang H, Calizo RC, Walsh JJ, et al. KCNQ channel openers reverse depressive symptoms via an active resilience mechanism. Nat Commun 2016, 7: 11671.

26. Bennett GJ, Xie YK. A peripheral mononeuropathy in rat that produces disorders of pain sensation like those seen in man. Pain 1988, 33: 87-107.

27. Hargreaves K, Dubner R, Brown F, Flores C, Joris J. A new and sensitive method for measuring thermal nociception in cutaneous hyperalgesia. Pain 1988, 32: 77-88.

28. Brown DA, Passmore GM. Neural KCNQ (Kv7) channels. Br J Pharmacol 2009, 156: 1185-1195. 
29. Brown DA, Adams PR. Muscarinic suppression of a novel voltage-sensitive $\mathrm{K}+$ current in a vertebrate neurone. Nature 1980, 283: 673-676.

30. Koyama S, Appel SB. Characterization of M-current in ventral tegmental area dopamine neurons. J Neurophysiol 2006, 96: 535-543.

31. Wang HS, Pan Z, Shi W, Brown BS, Wymore RS, Cohen IS, et al. KCNQ2 and KCNQ3 potassium channel subunits: molecular correlates of the M-channel. Science 1998, 282: 1890-1893.

32. Cooper EC, Harrington E, Jan YN, Jan LY. M channel KCNQ2 subunits are localized to key sites for control of neuronal network oscillations and synchronization in mouse brain. J Neurosci 2001, 21: 9529-9540.

33. Dalby-Brown W, Hansen HH, Korsgaard MP, Mirza N, Olesen SP. K(v)7 channels: function, pharmacology and channel modulators. Curr Top Med Chem 2006, 6: 999-1023.

34. Weber YG, Geiger J, Kampchen K, Landwehrmeyer B, Sommer $\mathrm{C}$, Lerche $\mathrm{H}$. Immunohistochemical analysis of KCNQ2 potassium channels in adult and developing mouse brain. Brain Res 2006, 1077: 1-6.

35. Main MJ, Cryan JE, Dupere JR, Cox B, Clare JJ, Burbidge SA. Modulation of $\mathrm{KCNQ} 2 / 3$ potassium channels by the novel anticonvulsant retigabine. Mol Pharmacol 2000, 58: 253-262.

36. Wuttke TV, Seebohm G, Bail S, Maljevic S, Lerche H. The new anticonvulsant retigabine favors voltage-dependent opening of the Kv7.2 (KCNQ2) channel by binding to its activation gate. Mol Pharmacol 2005, 67: 1009-1017.

37. Porter RJ, Nohria V, Rundfeldt C. Retigabine. Neurotherapeutics 2007, 4: 149-154.

38. Baquet ZC, Bickford PC, Jones KR. Brain-derived neurotrophic factor is required for the establishment of the proper number of dopaminergic neurons in the substantia nigra pars compacta. J Neurosci 2005, 25: 6251-6259.

39. Cohen-Cory S, Kidane AH, Shirkey NJ, Marshak S. Brainderived neurotrophic factor and the development of structural neuronal connectivity. Dev Neurobiol 2010, 70: 271-288.

40. Xia SH, Hu SW, Ge DG, Liu D, Wang D, Zhang S, et al. Chronic pain impairs memory formation via disruption of neurogenesis mediated by mesohippocampal BDNF signaling. Biol Psychiatry 2020, 88: 597-610.

41. Morales M, Margolis EB. Ventral tegmental area: cellular heterogeneity, connectivity and behaviour. Nat Rev Neurosci 2017, 18: 73-85.

42. Lammel S, Hetzel A, Hackel O, Jones I, Liss B, Roeper J. Unique properties of mesoprefrontal neurons within a dual mesocorticolimbic dopamine system. Neuron 2008, 57: 760-773.

43. Juarez B, Han MH. Diversity of dopaminergic neural circuits in response to drug exposure. Neuropsychopharmacology 2016, 41: 2424-2446.

44. Mellor D, Cook C, Stafford K. Quantifying some responses to pain as a stressor. In: The biology of animal stress: basic principles and implications for welfare. $1^{\text {st }}$ ed. CABI Publishing, 2000: 171-198.

45. Chen LM. Cortical representation of pain and touch: evidence from combined functional neuroimaging and electrophysiology in non-human primates. Neurosci bull 2018, 34: 165-177.

46. Lammel S, Lim BK, Malenka RC. Reward and aversion in a heterogeneous midbrain dopamine system. Neuropharmacology 2014, 76: 351-359.

47. Walsh JJ, Han MH. The heterogeneity of ventral tegmental area neurons: Projection functions in a mood-related context. Neuroscience 2014, 282: 101-108.
48. Han MH, Russo SJ, Nestler EJ. Molecular, Cellular, and Circuit Basis of Depression Susceptibility and Resilience. In: Neurobiology of depression: road to novel therapeutics. $1^{\text {st }}$ ed. San Diego: Academic Press. 2019: 123-136.

49. Douma EH, de Kloet ER. Stress-induced plasticity and functioning of ventral tegmental dopamine neurons. Neurosci Biobehav Rev 2020, 108: 48-77.

50. Chaudhury D, Walsh JJ, Friedman AK, Juarez B, Ku SM, Koo $\mathrm{JW}$, et al. Rapid regulation of depression-related behaviours by control of midbrain dopamine neurons. Nature 2013, 493: 532-536.

51. Liu D, Tang QQ, Yin C, Song Y, Liu Y, Yang JX, et al. Brainderived neurotrophic factor-mediated projection-specific regulation of depressive-like and nociceptive behaviors in the mesolimbic reward circuitry. Pain 2018, 159: 175.

52. Huang S, Borgland SL, Zamponi GW. Dopaminergic modulation of pain signals in the medial prefrontal cortex: Challenges and perspectives. Neurosci Lett 2019, 702: 71-76.

53. Wladyka CL, Kunze DL. KCNQ/M-currents contribute to the resting membrane potential in rat visceral sensory neurons. J Physiol 2006, 575: 175-189.

54. Rivera-Arconada I, Martinez-Gomez J, Lopez-Garcia JA. M-current modulators alter rat spinal nociceptive transmission: an electrophysiological study in vitro. Neuropharmacology 2004, 46: 598-606.

55. Friedman AK, Walsh JJ, Juarez B, Ku SM, Chaudhury D, Wang $\mathrm{J}$, et al. Enhancing depression mechanisms in midbrain dopamine neurons achieves homeostatic resilience. Science 2014, 344: 313-319.

56. Bal M, Zhang J, Zaika O, Hernandez CC, Shapiro MS. Homomeric and heteromeric assembly of KCNQ (Kv7) $\mathrm{K}^{+}$ channels assayed by total internal reflection fluorescence/fluorescence resonance energy transfer and patch clamp analysis. J Biol Chem 2008, 283: 30668-30676.

57. Jentsch TJ. Neuronal KCNQ potassium channels: physiology and role in disease. Nat Rev Neurosci 2000, 1: 21-30.

58. Hansen HH, Ebbesen C, Mathiesen C, Weikop P, Ronn LC, Waroux $\mathrm{O}$, et al. The KCNQ channel opener retigabine inhibits the activity of mesencephalic dopaminergic systems of the rat. J Pharmacol Exp Ther 2006, 318: 1006-1019.

59. Morikawa H, Paladini CA. Dynamic regulation of midbrain dopamine neuron activity: intrinsic, synaptic, and plasticity mechanisms. Neuroscience 2011, 198: 95-111.

60. Neuhoff H, Neu A, Liss B, Roeper J. I(h) channels contribute to the different functional properties of identified dopaminergic subpopulations in the midbrain. J Neurosci 2002, 22: 1290-1302.

61. Seutin V, Massotte L, Renette MF, Dresse A. Evidence for a modulatory role of $\mathrm{Ih}$ on the firing of a subgroup of midbrain dopamine neurons. Neuroreport 2001, 12: 255-258.

62. Zolles G, Klocker N, Wenzel D, Weisser-Thomas J, Fleischmann $\mathrm{BK}$, Roeper J, et al. Pacemaking by $\mathrm{HCN}$ channels requires interaction with phosphoinositides. Neuron 2006, 52: 1027-1036

63. Koyama S, Brodie MS, Appel SB. Ethanol inhibition of $\mathrm{m}$-current and ethanol-induced direct excitation of ventral tegmental area dopamine neurons. J Neurophysiol 2007, 97: 1977-1985.

64. Chu HY, Zhen X. Hyperpolarization-activated, cyclic nucleotidegated $(\mathrm{HCN})$ channels in the regulation of midbrain dopamine systems. Acta Pharmacol Sin 2010, 31: 1036-1043.

65. Wainger BJ, Kiskinis E, Mellin C, Wiskow O, Han SS, Sandoe J, et al. Intrinsic membrane hyperexcitability of amyotrophic lateral sclerosis patient-derived motor neurons. Cell Rep 2014, 7: 1-11. 
66. Knapp CM, O'Malley M, Datta S, Ciraulo DA. The Kv7 potassium channel activator retigabine decreases alcohol consumption in rats. Am J Drug Alcohol Abuse 2014, 40: 244-250.

67. Cerina M, Szkudlarek HJ, Coulon P, Meuth P, Kanyshkova T, Nguyen $\mathrm{XV}$, et al. Thalamic Kv 7 channels: pharmacological properties and activity control during noxious signal processing. Br J Pharmacol 2015, 172: 3126-3140.

68. Xu W, Wu Y, Bi Y, Tan L, Gan Y, Wang K. Activation of voltage-gated $\mathrm{KCNQ} / \mathrm{Kv} 7$ channels by anticonvulsant retigabine attenuates mechanical allodynia of inflammatory temporomandibular joint in rats. Mol Pain 2010, 6: 49. 